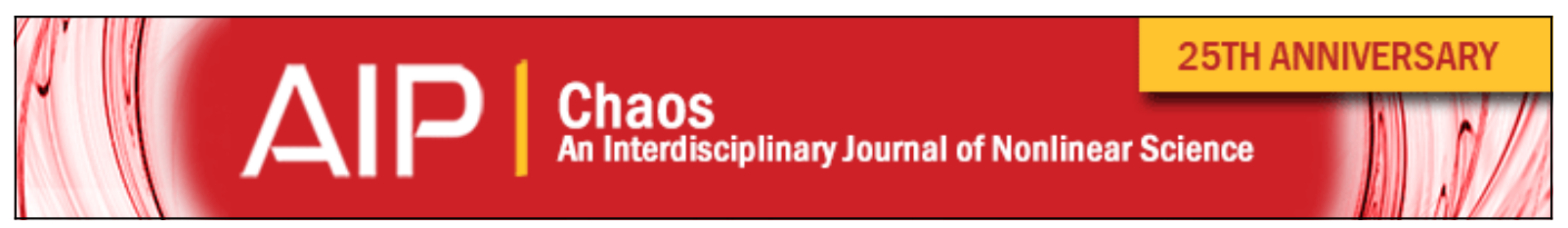

\title{
Dual-lag synchronization between coupled chaotic lasers due to path-delay interference
}

J. Tiana-Alsina, J. H. Garcia-Lopez, M. C. Torrent, and J. Garcia-Ojalvo

Citation: Chaos 21, 043102 (2011); doi: 10.1063/1.3644392

View online: http://dx.doi.org/10.1063/1.3644392

View Table of Contents: http://scitation.aip.org/content/aip/journal/chaos/21/4?ver=pdfcov

Published by the AIP Publishing

\section{Articles you may be interested in}

Simple adaptive output-feedback lag-synchronization of multiple time-delayed chaotic systems

Chaos 22, 023145 (2012); 10.1063/1.4729140

Synchronization and chaotic dynamics of coupled mechanical metronomes

Chaos 19, 043120 (2009); 10.1063/1.3266924

Projective-anticipating, projective, and projective-lag synchronization of time-delayed chaotic systems on random networks

Chaos 18, 023117 (2008); 10.1063/1.2912720

A new scheme to generalized (lag, anticipated, and complete) synchronization in chaotic and hyperchaotic systems

Chaos 15, 013101 (2005); 10.1063/1.1827431

Role of unstable periodic orbits in phase and lag synchronization between coupled chaotic oscillators

Chaos 13, 309 (2003); 10.1063/1.1518430

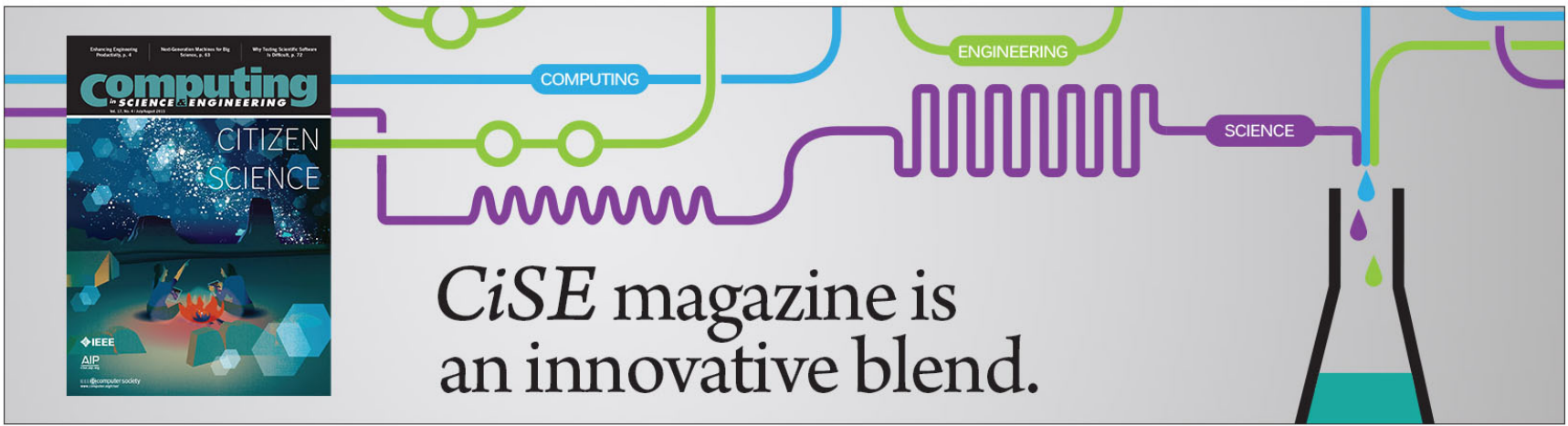




\title{
Dual-lag synchronization between coupled chaotic lasers due to path-delay interference
}

\author{
J. Tiana-Alsina, ${ }^{1}$ J. H. Garcia-Lopez, ${ }^{2}$ M. C. Torrent, ${ }^{1}$ and J. Garcia-Ojalvo ${ }^{1}$ \\ ${ }^{1}$ Departament de Fisica i Enginyeria Nuclear, Universitat Politècnica de Catalunya, Edifici GAIA, \\ Rambla de Sant Nebridi s/n, 08222 Terrassa, Spain \\ ${ }^{2}$ Universidad de Guadalajara, Centro Universitario de los Lagos, Enrique Díaz de Leon, \\ Paseos de la Montaña, 47460 Lagos de Moreno, Jalisco, Mexico
}

(Received 2 July 2011; accepted 9 September 2011; published online 3 October 2011)

\begin{abstract}
We study experimentally the synchronization dynamics of two semiconductor lasers coupled unidirectionally via two different delayed paths. The emitter laser operates in a chaotic regime characterized by low-frequency fluctuations due to optical feedback and induces a synchronized dynamical activity in the receiver laser, which operates in the continuous-wave regime when uncoupled. Different delays in the two coupling paths lead to the coexistence of two time lags in the synchronized dynamics of the oscillators. This dual-lag synchronization degrades the average synchronization quality of the system of coupled lasers and hinders the transmission of information between them. Numerical simulation results agree with the experimental observations, and allow us to explore this phenomenon in a wide parameter range, and quantify the degree of signal transmission degradation caused by this chaotic path-delay interference. () 2011 American Institute of Physics. [doi:10.1063/1.3644392]
\end{abstract}

Complex systems such as the brain, the World-Wide Web, and the electrical grid are information-processing networks in which signals travel through a tangled mesh of connections that link dynamical nodes. ${ }^{1,2}$ Under these conditions, information between two given elements of the network usually travels simultaneously along multiple paths. This situation is very different from the classical scenario considered traditionally in chaos communications, in which two chaotic oscillators are usually coupled through a single communication channel..$^{3-5}$ Moreover, in most situations, the time taken by the coupling signal to travel along any given path is comparable to, or even larger than, the characteristic time of the nodes' dynamics. In that case, coupling delay times cannot be ignored, leading at best (in the case of single-path coupling) to lag synchronization between the emitter and the receiver. When the coupling delay times along the various coupling paths differ (which is the most common situation in complex networks ${ }^{6}$ ), interference between the associated lag times can be expected to affect the synchronization dynamics and thus the communication efficiency. Here, we study experimentally this phenomenon in a simplified setting, using as a model system two semiconductor lasers coupled optically via two paths of different lengths. When the coupling strengths along the two paths are comparable, the synchronization lags corresponding to the two paths are seen to coexist in the synchronized dynamics. The dependence of this phenomenon on the coupling ratio is studied, and its impact on the chaos communication efficiency, is examined.

\section{INTRODUCTION}

Semiconductor lasers are excellent model systems for nonlinear dynamical studies, because they can be easily con- trolled experimentally, theoretical models exist that reproduce closely their behavior, and they exhibit a wide variety of dynamical regimes when perturbed by optical injection, modulation of their pump current, or feedback. ${ }^{7}$ Coherent optical feedback, in particular, is well known to induce fully developed chaotic dynamics in the laser (known as coherence collapse), and also a highly structured chaotic regime consisting of sudden intensity dropouts from an otherwise stable emission state, followed by a slower recovery. ${ }^{8}$ This latter regime occurs over time scales of tens to hundreds of nanoseconds, much larger than the characteristic time of the laser itself, and is thus termed low-frequency-fluctuation (LFF) regime. ${ }^{9}$ This is the dynamical scenario that will be used in what follows.

Another characteristic of semiconductor lasers that makes them highly attractive for dynamical system studies is the natural manner in which they can be coupled to one another, forming networks of interacting dynamical elements. Coupling can be accomplished optoelectronically, by modulating the pump current of a laser with the electrical signal of a photodetector that measures the light emitted by another laser, ${ }^{10-12}$ or more simply in a purely optical manner, by injection (coherent or incoherent) of a laser's output light into another. ${ }^{13-15}$ Given the fast temporal scales (on the order of picoseconds) with which semiconductor lasers operate, the time taken by the coupling signals to travel between the lasers (typically larger than nanoseconds) cannot be usually ignored, and thus interacting lasers become instances of delay-coupled dynamical systems. ${ }^{16}$ Within that context, a unidirectional coupling configuration in which the light emitted by a chaotic laser is injected into a second laser (which is stable in the absence of injection) readily exhibits synchronization with a time lag equal to the coupling delay time, in such a way that the emitting laser leads over the receiver. ${ }^{17}$ 


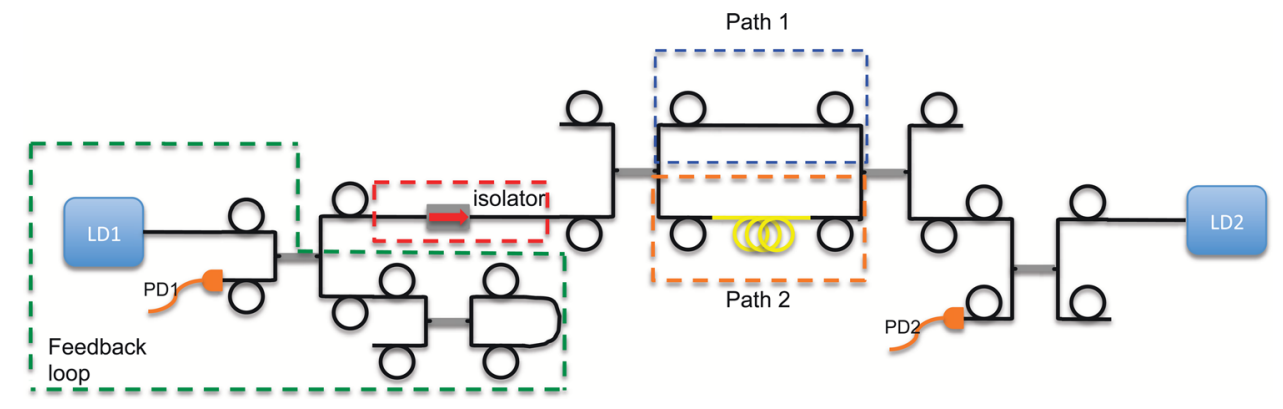

FIG. 1. (Color online) Experimental setup of two unidirectionally coupled semiconductor lasers via two paths of different lengths. LD1 is the emitter laser and receives optical feedback via a closed loop. LD2 is the receiver laser. PD1 and PD2 are photodetectors, and the optical isolator ensures unidirectionality. The thick grey segments represent optical couplers and black lines denote optical fibers, with the multiple loops in path 2 (yellow online) depicting extra fiber added to that path to increase its length with respect to path 1 .

Two semiconductor lasers bidirectionally coupled via mutual injection of their emitted light fields also synchronize their dynamics in a generalized way (which for bias currents close to threshold can take the form of low-frequency fluctuations induced by the coupling itself) with a lag time corresponding to the coupling delay time. In this case, the sign of the lag depends on the relative frequencies of the lasers: the device with higher frequency leads the dynamics, whereas if the two lasers have the same frequency, the sign of the lag alternates randomly in time, in what constitutes a spontaneous symmetry breaking. ${ }^{18}$ This random switching is known to impair substantially the ability of the coupled laser system to transmit information. ${ }^{19}$ Isochronal synchronization can be obtained if the coupling time is tuned adequately..$^{20,21}$

Few studies exist so far involving more than two lasers coupled optically. Three-laser systems have been shown experimentally to undergo a route to synchronization via clustering, ${ }^{22}$ while theoretical studies have addressed the collective behavior of higher numbers of coupled lasers. ${ }^{23,24}$ The tendency is to move towards larger systems that can be used as experimental models of complex networks of dynamical elements. ${ }^{1}$ An outstanding issue in these networks is how information is transmitted between pairs of nodes. Usually multiple paths exist connecting a given pair of nodes, and thus (if the speed of the coupling signals cannot be neglected) multiple coupling delays are involved in the communication between them. In those conditions, it is not clear what type of synchronized dynamics we should expect: Will one of the two coupling delays dominate and lead to a consistent lag between the lasers? If so, under what conditions will that happen? And what will be the dynamics when none of the two delays is dominant? Will the synchronization lag alternate irregularly between the two delays, similarly to the lag alternation found between two mutually coupled lasers? ? $^{18,19}$

In order to address these questions, here, we study experimentally a minimal model system consisting of two optically coupled semiconductor lasers connected unidirectionally via two distinct paths, with different coupling delays. The emitter laser is led to operate in the chaotic lowfrequency-fluctuation regime, due to optical feedback. This chaotic dynamics is transmitted simultaneously down the two coupling paths towards the other laser, which operates in a continuous-wave regime when uncoupled from the emitter laser. Injection from the emitter leads to chaotic dynamics in the receiver, synchronized with that of the emitter with a lag that depends on the coupling conditions. When one of the two coupling paths prevails upon the other, in terms of the relative amount of light travelling through them, the delay time associated to the prevailing path dominates, becoming the synchronization lag between the dynamics of emitter and receiver. On the other hand, when no path dominates over the other, a state of dual-lag synchronization emerges in which the two coupling delays coexist in the synchronized dynamics of the two lasers.

Our experimental setup allows us to systematically investigate these regime of dual-lag synchronization as a function of the relative coupling strength. Numerical simulations of a delay-differential model further allow us to examine the phenomenon in a wide parameter regime and interpret its existence. Finally, the influence of this lag coexistence on the ability of the system to transmit information is examined both experimentally and numerically. The paper is organized as follows. Section II contains a description of the experimental setup. The experimental results obtained are shown in Sec. III, followed by a description of the theoretical model and its simulation in Sec. IV. Finally, the ability of the system to transmit information in the presence of two distinct communication paths is discussed in Sec. V.

\section{EXPERIMENTAL SETUP}

A schematic diagram of the experimental setup is shown in Fig. 1. Two semiconductor lasers (Mitsubishi ML925B45F) with a nominal wavelength $\lambda_{n}=1550 \mathrm{~nm}$ are coupled unidirectionally, with LD1 being the emitter and LD2 the receiver. The temperature and pump current of the lasers are controlled with an accuracy of $0.01{ }^{\circ} \mathrm{C}$ and 0.01 $\mathrm{mA}$, respectively, and are adjusted such that the optical frequencies of LD1 (with its feedback; see below) and LD2 (with injection) are as similar as possible to each other. For temperatures $T_{L D 1}=19.91^{\circ} \mathrm{C}$ and $T_{L D 2}=19.99^{\circ} \mathrm{C}$, the threshold currents of the solitary lasers are, respectively, $I_{L D 1}^{\text {th }}=11.10 \mathrm{~mA}$ and $I_{L D 2}^{\text {th }}=11.63 \mathrm{~mA}$.

The laser LD1 is subject to delayed feedback from the closed fiber loop shown at the left side of Fig. 1, whose length $(10 \mathrm{~m})$ provides for a feedback time of $55 \mathrm{~ns}$. The output of this laser is injected, in a unidirectional coupling configuration, into laser LD2 via two different paths with two 
distinct delays, with $\tau_{1}=65 \mathrm{~ns}$ for path 1 and $\tau_{2}=97 \mathrm{~ns}$ for path 2 , corresponding to fiber lengths of 12 and $18 \mathrm{~m}$, respectively. In order to control the relative influence of the couplings, we introduce variable optical attenuators in the two paths. The reduction of the threshold current of laser LD1 due to its feedback is $4.2 \%$. The reduction of the threshold current of laser LD2 due to the injection through path 2 is $0.89 \%$, and the one due to injection through path 1 ranges from $1.86 \%$ to $0.05 \%$. The laser intensities are captured by high-speed fiber photodetectors with a bandwidth of $2 \mathrm{GHz}$ (DET01CFC). The electrical signals received are amplified, using $2 \mathrm{GHz}$ high-speed amplifiers (Femto), and sent to a 1-GHz-bandwidth oscilloscope (Agilent DS06104A) and a power spectrum analyzer (Anritsu MS2651B). Due to the relatively small bandwidth of the detectors, we are only able to show the slower dynamics of the system, which is enough for our study.

\section{EXPERIMENTAL RESULTS}

Due to the delayed feedback mentioned above, the laser LD1 oscillates chaotically in a regime of low-frequency fluctuations. This chaotic behavior is injected into LD2 via the two different paths described in Sec. II. In those conditions, synchronization between the two lasers can be achieved when one or both paths are switched on, provided the transmission strength is large enough. In order to characterize the quality of synchronization between emitter and receiver, and to determine which coupling delay time (if any) dominates the synchronized dynamics, we calculate the cross-correlation function between the (filtered) time series of the light detected by the two photodiodes,

$$
C(\Delta t)=\frac{\left\langle\left[I_{1}(t)-\left\langle I_{1}\right\rangle\right]\left[I_{2}(t+\Delta t)-\left\langle I_{2}\right\rangle\right]\right\rangle}{\sqrt{\left\langle\left[I_{1}(t)-\left\langle I_{1}\right\rangle\right]^{2}\right\rangle\left\langle\left[I_{2}(t)-\left\langle I_{2}\right\rangle\right]^{2}\right\rangle}} .
$$

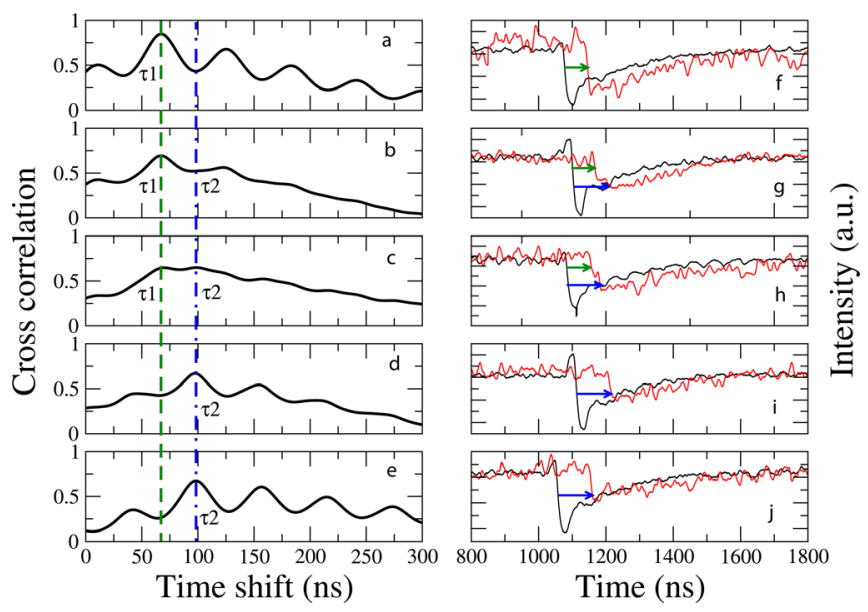

FIG. 2. (Color online) Experimental cross-correlation function between LD1 and LD2 (left column) and corresponding time traces of the output intensities (right column) for different values of coupling in the two paths. In panels (a) and (f) only path 1 is switched on, while in panels (e) and (j) only path 2 is activated. The attenuation of path 2 is constant and equal to 5 $\mathrm{dB}$ in panels (b)-(e) and (g)-(j), while the attenuation of path 1 is $5 \mathrm{~dB}$ in panels (a) and (f) and (b) and (g), $10 \mathrm{~dB}$ in panels (c) and (h), and $15 \mathrm{~dB}$ in panels (d) and (i).
The left plots in Fig. 2 show the cross-correlation function between the two laser outputs for varying levels of coupling in the two paths. Figure 2(a) corresponds to the case where path 2 is blocked; accordingly, the highest cross-correlation peak occurs at $\tau_{1}$, which means that laser LD1 leads LD2 with a lag equal to $\tau_{1}$. In plots (b) to (e) the path 2 is open with a relatively low attenuation, while the attenuation of path 1 is progressively increased, until that path is completely blocked in Fig. 2(e). Correspondingly, a peak in the crosscorrelation function at $\tau_{2}$ (which is the the flight time along path 2) appears and eventually dominates in the crosscorrelation function. For an intermediate value of the attenuation $[10 \mathrm{~dB}$, Fig. 2(c)] the two paths are approximately equivalent and the cross-correlation function exhibits two coexisting global maxima at $\tau_{1}$ and $\tau_{2}$, separated by a shallow valley whose small relative depth is a consequence of the filtering of the time series, as we can verify from simulations of the theoretical model described below. In this regime, no single coupling delay dominates over the other, but they both coexist in the synchronized dynamics of the system. Note that the value of the cross-correlation at this two time lags is on the order of 0.75 , corresponding to a significant level of synchronization (in the two single-path limits-plots (a) and (e) - the cross-correlation scarcely reaches 0.80 ).

In order to establish the nature of the lag-coexistence scenario revealed in Fig. 2(c) (in particular, to determine if this regime corresponds to a lag alternation similar to that occurring in two mutually-coupled lasers ${ }^{18}$ ), we plot in the right panels of Fig. 2 the time series of the two laser intensities. When only one of the paths is switched on [Figs. 2(a), 2(f) and 2(e), 2(j)], the dropouts exhibit the usual single-lag synchronization dynamics, in which LD2 follows LD1 with a lag $\tau_{1}$ or $\tau_{2}$ depending on which one is the dominating path. This behavior, with a clear delay of $\tau_{1}$ or $\tau_{2}$ in the dropouts of LD2 after those of LD1, even when the two paths are open, provided that one path dominates over the other [Figs. 2(b), 2(g) and 2(d), 2(i)]. On the other hand, in the transition between the $\tau_{1}$ - and $\tau_{2}$-dominated regimes, during which the two lags coexist in the cross-correlation function [Fig. 2(c)], the dropouts of LD2 are qualitatively different. In principle, one could expect that in this lag-coexistence regime, a fraction of the LD2 dropouts would follow LD1 with a delay $\tau_{1}$, and the rest would follow LD1 with delay $\tau_{2}$. This, however, is not what happens, as shown in the time series plotted in Figs. 2(g) and 2(h). In this figure, the dropout of LD2 (red line) consists of a first fall in intensity that follows LD1 with a delay $\tau_{1}$, after which the intensity of LD2 remains approximately constant for a short instant, until finally there is a second fall in intensity that follows the original LD1 dropout with a delay $\tau_{2}$. This behavior is consistent for all LD2 dropouts. Thus, although the cross-correlation function is here similar to the case of two bidirectionally coupled lasers, which also has two peaks, this does not imply that the dynamical behavior is the same. In the case of bidirectional coupling, the two peaks in the cross-correlation imply a (random) alternation in time between the leader and laggard role of the dynamics, and not a modification of the shape of the dropouts, as happens in the dual-lag synchronization regime occurring in this case. 


\section{NUMERICAL SIMULATIONS}

In order to verify the nature of the dual-lag synchronization regime described above, it would be helpful to increase the difference in the two coupling paths, which should increase the duration of the plateau between the two falls in the intensity of LD2 that we interpret to be occurring during the LD2 dropouts, and which would give rise to the lagcoexistence observed in the cross-correlation function. However, technical reasons prevented to vary in a systematic manner the delay times, since laser coupling was made by optical fibers, and we would need to have a sufficiently large number of fibers of increasing length. In order to circumvent this requirement, we resorted to numerical simulations of the system, which is known to be accurately described by the Lang-Kobayashi model for moderate feedback strengths and single-mode operation (conditions which approximately hold in our experiment). The generalized Lang-Kobayashi model that we use is the following: ${ }^{19}$

$$
\begin{aligned}
\frac{d E_{1}}{d t}= & \frac{1+i \alpha}{2}\left(G_{1}-\gamma_{1}\right) E_{1}(t)+\kappa_{f} e^{-i \omega_{1} \tau_{f}} E_{1}\left(t-\tau_{f}\right) \\
& +\sqrt{2 \beta N_{1}} \xi_{1}(t) \\
\frac{d E_{2}}{d t}= & \frac{1+i \alpha}{2}\left(G_{2}-\gamma_{2}\right) E_{2}(t)+\sum_{j} \kappa_{j} e^{i\left(\Delta \omega t-\omega_{j} \tau_{j}\right)} E_{1}\left(t-\tau_{j}\right) \\
& +\sqrt{2 \beta N_{2}} \xi_{2}(t) \\
& \frac{d N_{i}}{d t}=C_{b}-\gamma_{e} N_{t h}-G_{i} I_{i}(t)
\end{aligned}
$$

where subindex $i=1,2$ denotes lasers LD1 and LD2, respectively, while subindex $j=1,2$ represents each of the two paths going from LD1 to LD2. $E_{i}(t)$ is the electric field and $I_{i}(t)=\left|E_{i}(t)\right|^{2}$ is the intensity of each laser, while $N_{i}$ is the corresponding carrier number and $\omega_{i}$ the free-running optical frequency. Here, we consider a detuning $\Delta \omega=\omega_{1}-\omega_{2}$ $=15.89 \mathrm{GHz}$ between the lasers, with $\omega_{1}$ being the frequency corresponding exactly to $\lambda=1550 \mathrm{~nm}$. The laser gain is given by $G_{i}=g\left(N_{i}-N_{0}\right)$ (assumed linear), where $N_{0}$ denotes the carrier number at transparency and $g$ is the differential gain, both of which are assumed equal for the two lasers (gain saturation is neglected because the lasers operate close to threshold). $\gamma$ represents the inverse photon lifetime and $\alpha$ the linewidth enhancement factor; both are also assumed to be the same for the two lasers. The second term in the right-hand side of Eq. (2) is the feedback term, with associated feedback delay time $\tau_{f}$ and feedback strength $\kappa_{f}$. The second term in Eq. (3) is the coupling term, with coupling delay times $\tau_{j}$ and coupling strengths $\kappa_{j}$, for the two paths $j=1,2$. The last term in these two equations corresponds to the spontaneous emission noise, represented by a Gaussian white noise $\xi_{i}(t)$ of zero mean, with spontaneous emission rate $\beta$. The carrier density Eq. (4) contains three terms. The first one is the bias current corresponding to the injection current of each laser (defined as $C_{b}=\gamma_{e} N_{t h} \frac{I_{p}}{I_{t h}}$, where $N_{t h}=\frac{\gamma}{g}+N_{0}$, and the pump current relative to threshold, $I_{p} / I_{t h}$, is also assumed equal for the two lasers).
TABLE I. Laser parameters of the numerical model.

\begin{tabular}{lcc}
\hline \hline Symbol & Parameter & Value \\
\hline$I_{p}$ & Pump current of LD1,2 & $1.01 \cdot I_{\text {th }}$ \\
$T_{1}$ & Coupling time path 1 & $65 \mathrm{~ns}$ \\
$T_{2}$ & Coupling time path 2 & $97 \mathrm{~ns}$ \\
$\tau_{f}$ & Feedback time & $55 \mathrm{~ns}$ \\
$\gamma_{e}$ & Inverse carrier lifetime & $6.89 \cdot 10^{-4} \mathrm{~ns}^{-1}$ \\
$\gamma$ & Inverse photon lifetime & $0.480 \mathrm{ps}^{-1}$ \\
$N_{0}$ & Carrier number at transparency & $1.25 \cdot 10^{8}$ \\
$N_{t h}$ & Carrier number at threshold & $1.634 \cdot 10^{8}$ \\
$g$ & Gain parameter & $1.25 \cdot 10^{-8} \mathrm{ps}^{-1}$ \\
$\alpha$ & Linewidth enhancement factor & 4.0 \\
$\beta$ & Spontaneous emission rate & $1 \cdot 10^{-15} \mathrm{ps}^{-1}$ \\
\hline \hline
\end{tabular}

The parameters used in the numerical simulations are given in Table I. For these parameters, the emitter laser operates in the LFF regime. Figures 3(a)-3(e) shows the numerically computed cross-correlation functions between the filtered time series of the lasers, and Figs. 3(f)-3(j) reproduce the corresponding time traces. In order to simulate the experimental filter (due to the relatively small bandwidth of our experimental setup) we filter the output intensities with a fourth-order Butterworth filter with a cutoff frequency of $100 \mathrm{MHz}$. The numerical results displayed in the figure qualitatively reproduce the experimental results. In particular, the simulations show that in the dual-lag synchronization regime [Figs. 3(c) and 3(h)], the LD2 dropouts consist of two intensity falls occurring at times $\tau_{1}$ and $\tau_{2}$ after the corresponding LD1 dropout, separated by a plateau. Numerical simulations also allow us to examine the dynamics at an arbitrary temporal resolution. In the absence of filtering, the crosscorrelation function exhibits more clearly defined peaks, but the dual-lag synchronization behavior is not evident in the unfiltered time series (results not shown).
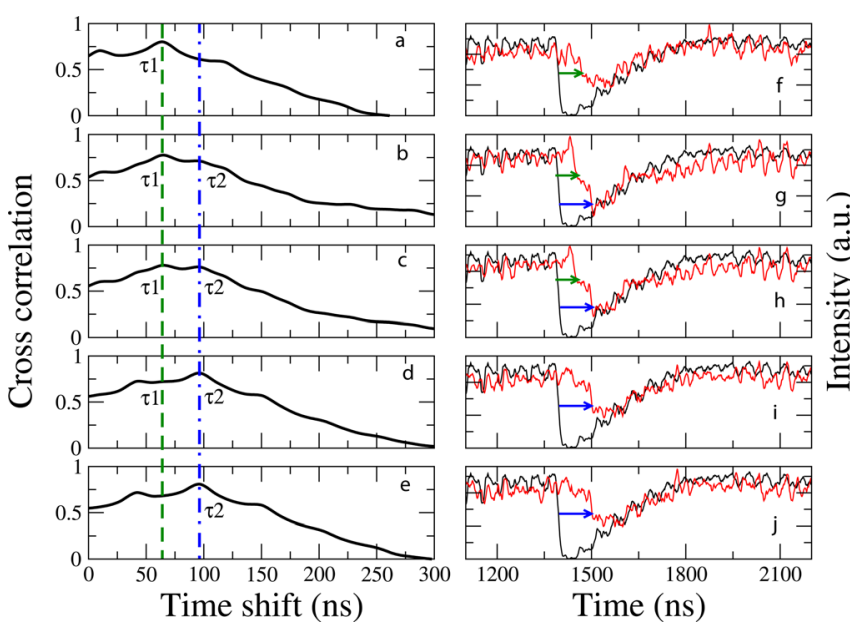

FIG. 3. (Color online) Numerical cross-correlation function between LD1 and LD2 (left column) and the corresponding output intensities (right column) for different values of the coupling strengths: (a)and (f) $\kappa_{1}=0 \mathrm{~ns}^{-1}$, $\kappa_{2}=40 \mathrm{~ns}^{-1}$ (a) and (f), (b) and (g) $\kappa_{1}=20 \mathrm{~ns}^{-1}, \kappa_{2}=40 \mathrm{~ns}^{-1}$, (c) and (h) $\kappa_{1}=40 \mathrm{~ns}^{-1}, \kappa_{2}=40 \mathrm{~ns}^{-1}$, (d) and (i) $\kappa_{1}=60 \mathrm{~ns}^{-1}, \kappa_{2}=40 \mathrm{~ns}^{-1}$, and (e) and $(\mathrm{j}) \kappa_{1}=40 \mathrm{~ns}^{-1}, \kappa_{2}=0 \mathrm{~ns}^{-1}$ (e) and (j). The feedback strength is fixed at $\kappa_{f}=60 \mathrm{~ns}^{-1}$. Other parameters are given in Table I. 


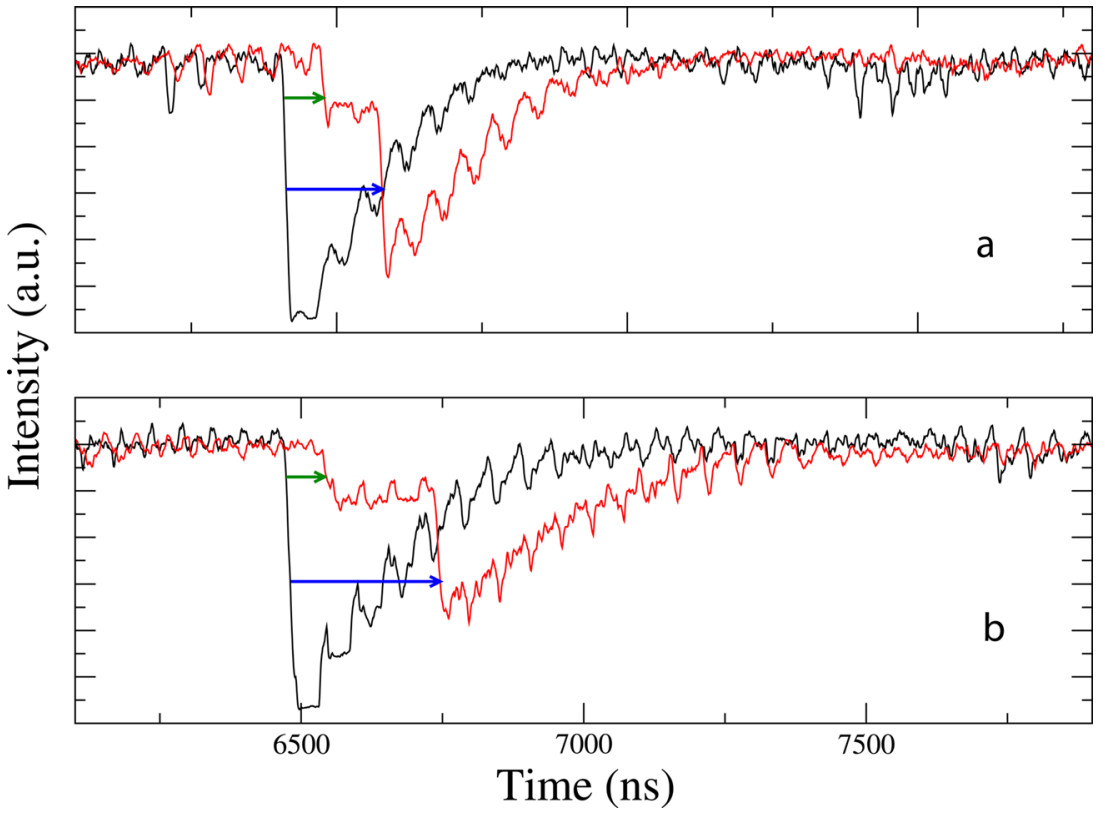

FIG. 4. (Color online) Numerical output intensities for different coupling times in path 2 . The delay time is $165 \mathrm{~ns}$ in (a) and is $265 \mathrm{~ns}$ in (b). The coupling parameters are $\kappa_{1}=\kappa_{2}=40 \mathrm{~ns}-{ }^{1}$ and $\kappa_{f}=30 \mathrm{~ns}^{-1}$. The other parameters are given in Table I.
In order to verify that the plateau in the LD2 dropout shown in Fig. 3(h) is not an artifact, we increased substantially in the numerical model the difference between the two coupling delays. The result of this test is displayed in Fig. 4, which shows the time traces of a pair of corresponding dropouts for two different values of $\tau_{2}: 165 \mathrm{~ns}$ in plot (a) and 265 ns in plot (b), while $\tau_{1}=65 \mathrm{~ns}$ in the two cases. Note that when we increase the coupling time of path 2 , the plateau of the LD2 dropout increases correspondingly. Thus, these simulations show clearly that the dual-lag dynamics is due to combined dropouts in LD2, consisting of two falls at the two lag times separated by a plateau.

\section{CHAOTIC COMMUNICATIONS}

Many studies have addressed the transmission of information between two chaotic lasers coupled unidirectionally via a single path. In this section, we want to study the viability of chaotic communications when the coupling between the lasers is done via two different paths, in the light of the dual-lag synchronization scenario described above. First we ask if the experimental setup presented in Fig. 1 above is able to filter a periodic signal introduced in the emitter laser, even in the case of dual-lag dynamics. This filtering mechanism is called chaos-pass filtering. ${ }^{15,25}$ As shown in Fig. 5, when a periodic modulation of frequency $1.20 \mathrm{MHz}$ is applied to the pump current of the emitter laser, the receiver laser filters out the modulation, and no corresponding peak in the power spectrum of the laggard laser can be observed. Note that this experimental result is obtained in the dual-lag regime (i.e., both paths are equally synchronized).

Once we have seen that chaos-pass filtering is possible between two semiconductor lasers unidirectionally coupled through two lag times, we now ask for the viability of

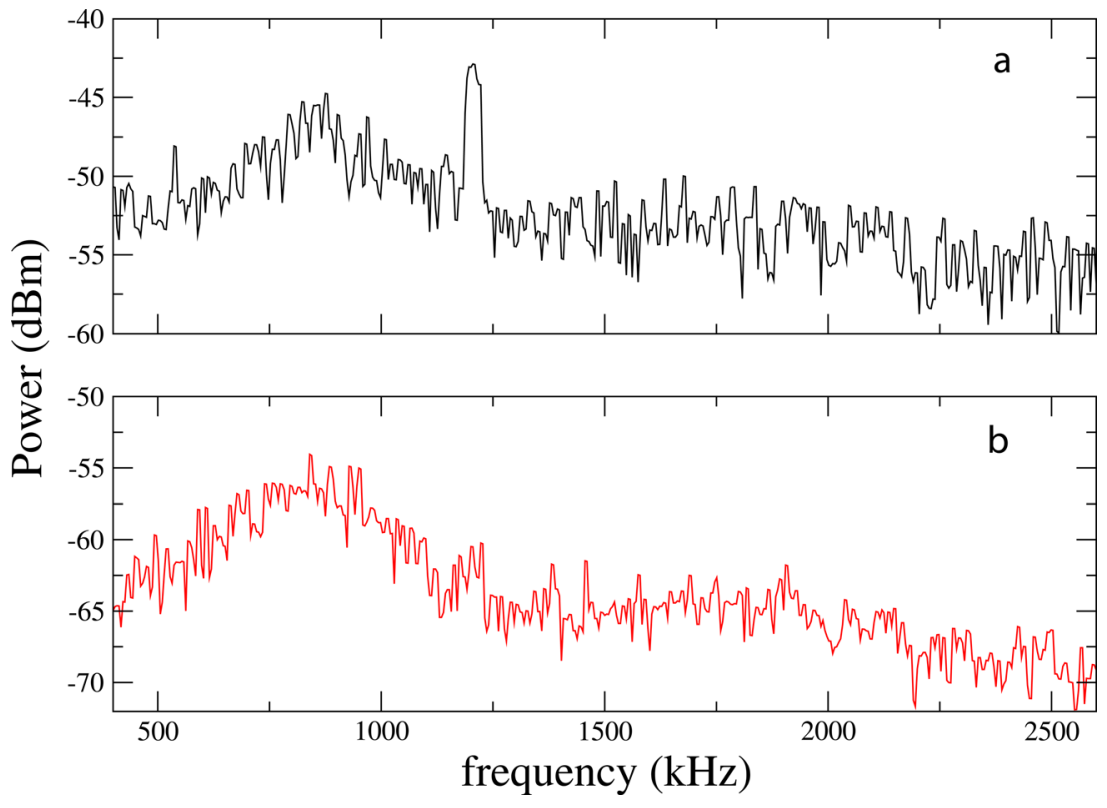

FIG. 5. (Color online) Spectral response of the receiver laser (b) to a modulation introduced in the emitter laser (a), in the case where both delays dominate equally the dynamics (dual-lag synchronization). 

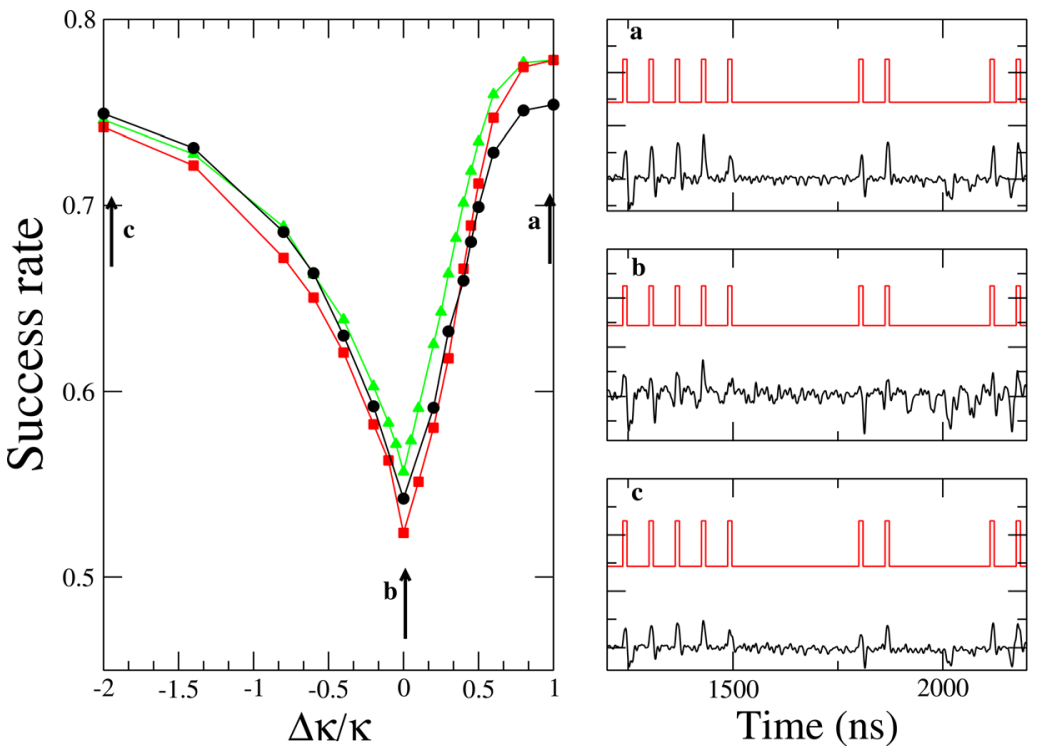

FIG. 6. (Color online) Left: Numerically computed success rate versus coupling asymmetry for three different pairs of coupling delay times, with $\tau_{2}=97 \mathrm{~ns}$ in all three cases, while $\tau_{1}$ equals $55 \mathrm{~ns}$ (squares), $65 \mathrm{~ns}$ (circles), and $75 \mathrm{~ns}$ (triangles). Right: input message (red) and recovered message (black) for three values of $\frac{\Delta \kappa}{\kappa}$, marked with arrows in the left plot (for the case $\tau_{2}=65 \mathrm{~ns}$ - circles-in the left panel). For the recovery, the time series in the receiver laser is shifted a time lag that corresponds to the delay of the path with strongest coupling. Parameters are those given in Table I, plus $\kappa_{2}=40 \mathrm{~ns}^{-1}$. recovering a message for the different setup configurations (i.e., only one path or both switched on). Figure 6 displays numerical results that evaluate the effectiveness of the message recovery for different values of $\frac{\Delta \kappa}{\kappa}=\frac{\kappa_{2}-\kappa_{1}}{\kappa_{2}}$, when a nonperiodic signal (specifically, a return-to-zero bit train) is introduced in the pump current of laser LD1. The message is introduced with an amplitude equal to $2.5 \%$ of the pump current and is decoded by subtracting the light emitted by the two lasers, taking into account the delay between the signals. The subtracted signal is filtered with a fourth-order Butterworth low-pass filter. In order to quantify the effectiveness of the recovered message, we calculate the success rate between the input and the output messages, as the fraction of bits are successfully recovered by LD2. To compute this quantity, the output message is binarized by defining a threshold, such that when the recovered message is larger than this threshold we assign a " 1 " and when it is smaller we assign a " 0 ." The left panel of Fig. 6 displays the success rate for varying values of the coupling asymmetry between the two paths, $\frac{\Delta \kappa}{\kappa}$, and for three different values of the difference between coupling delays. The maximum value of the success rate occurs for sufficiently large coupling strength asymmetry, which corresponds to a situation in which standard single-lag synchronization occurs, and the choice of time shift to be applied in the message recovery is simple. As we approach the dual-lag regime, on the other had, the success rate diminishes sharply. Thus, even though the receiver laser exhibits good chaos-pass filtering capabilities in the case of dual-lag synchronization, the coexistence of two lags in this case prevents the system from recovering the message. The time traces corresponding to three specific values of the coupling strength asymmetry (the two single-lag extremes and the dual-lag case) are shown in the right panels of Fig. 6. As expected, when the difference between the coupling delay times diminishes the success rate increases, because the interference effects degrading message recovery diminish.

\section{CONCLUSIONS}

We have examined the synchronized dynamics of two semiconductor lasers coupled unidirectionally via two different paths. The emitter laser operates in an intrinsically chaotic regime in the form of irregularly occurring power dropouts (low-frequency fluctuation regime), which generates chaotic behavior in the receiver. Our experimental results have revealed a transition a regime in which the synchronized dynamics is dominated by path 1 , and another one dominated by path 2 . In the transition region where both synchronization takes place via the two paths, two lags corresponding to the two coupling delays coexist, as shown by the crosscorrelation function. Our experiments and numerical simulations have shown that this dual-lag synchronization regime does not consist in an alternation between the two lags, as happens usually in mutually coupled semiconductor lasers, ${ }^{18,19}$ but in a dynamics through which the power dropouts of laser LD2 are formed by two falls in the laser intensity, occurring after the two coupling lags, separated by a plateau. This dynamical regimes does not prevent the receiver laser from performing chaos-pass filtering of an input modulation in the emitter laser's output but does prevent a successful recovery of the message. It would be interesting to extend this type of study first to the case of bidirectional coupling via two paths (where we can expect bidirectionality to interact in a nontrivial way with the path interference mechanism reported here), and second to the case of information propagation through complex networks, in which pairs of nodes are commonly coupled via multiple paths of different lengths, and thus with different coupling delays.

\section{ACKNOWLEDGMENTS}

We thank two anonymous reviewers for insightful comments on the initial version of this manuscript. Research supported by the Ministerio de Ciencia e Innovación (Spain, 
project FIS2009-13360) and by the ICREA Academia programme.

${ }^{1}$ S. Boccaletti, V. Latora, Y. Moreno, M. Chavez, and D. Hwang, Phys. Rep. 424, 175 (2006).

${ }^{2}$ A. Motter, M. Matias, J. Kurths, and E. Ott, Physica D 224, vii (2006).

${ }^{3}$ G. Vanwiggeren and R. Roy, Science 279, 1198 (1998).

${ }^{4}$ A. Uchida, F. Rogister, J. García-Ojalvo, and R. Roy, Prog. Opt. 48, 203 (2005).

${ }^{5}$ A. Argyris, D. Syvridis, L. Larger, V. Annovazzi-Lodi, P. Colet, I. Fischer, J. García-Ojalvo, C. Mirasso, L. Pesquera, and K. Shore, Nature (London) 438, 343 (2005).

${ }^{6}$ C. Masoller and A. C. Martí, Phys. Rev. Lett. 94, 134102 (2005).

${ }^{7}$ J. Sacher, D. Baums, P. Panknin, W. Elsässer, and E. Göbel, Phys. Rev. A 45, 1893 (1992).

${ }^{8}$ J. Ohtsubo, Prog. Opt. 44, 1 (2002).

${ }^{9}$ I. Fischer, G. Van Tartwijk, A. Levine, W. Elsässer, E. Göbel, and D. Lenstra, Phys. Rev. Lett. 76, 220 (1996).

${ }^{10}$ L. Larger, J. Goedgebuer, and F. Delorme, Phys. Rev. E 57, 6618 (1998).

${ }^{11}$ M. Kim, R. Roy, J. Aron, T. Carr, and I. Schwartz, Phys. Rev. Lett. 94, 88101 (2005).
${ }^{12}$ L. Illing, G. Hoth, L. Shareshian, and C. May, Phys. Rev. E 83, 026107 (2011).

${ }^{13}$ C. Mirasso, P. Colet, and P. García-Fernández, IEEE Photonics Technol. Lett. 8, 299 (1996).

${ }^{14}$ S. Sivaprakasam and K. Shore, Opt. Lett. 24, 1200 (1999).

${ }^{15}$ I. Fischer, Y. Liu, and P. Davis, Phys. Rev. A 62, 011801 (2000).

${ }^{16}$ H. Schuster and P. Wagner, Prog. Theor. Phys. 81, 939 (1989).

${ }^{17}$ Y. Takiguchi, H. Fujino, and J. Ohtsubo, Opt. Lett. 24, 1570-1572 (1999).

${ }^{18}$ T. Heil, I. Fischer, W. Elsässer, J. Mulet, and C. R. Mirasso, Phys. Rev. Lett. 86, 795 (2001).

${ }^{19}$ C. M. Gonzalez, M. C. Torrent, and J. Garcia-Ojalvo, Chaos 17, 033122 (2007).

${ }^{20}$ E. Klein, N. Gross, M. Rosenbluh, W. Kinzel, L. Khaykovich, and I. Kanter, Phys. Rev. E 73, 066214 (2006).

${ }^{21}$ J. M. Avila and J. R. Leite, Opt. Express 17, 21442 (2009).

${ }^{22}$ C. M. Gonzalez, C. Masoller, M. C. Torrent, and J. Garcia-Ojalvo, Europhys. Lett. 79, 64003 (2007).

${ }^{23}$ O. D'Huys, R. Vicente, T. Erneux, J. Danckaert, and I. Fischer, Chaos 18, 037116 (2008).

${ }^{24}$ J. Zamora-Munt, C. Masoller, J. Garcia-Ojalvo, and R. Roy, Phys. Rev. Lett. 105, 264101 (2010).

${ }^{25}$ A. Murakami and K. Shore, Phys. Rev. A 72, 053810 (2005). 